\title{
Practice Patterns in Gynecology Oncology
}

\author{
Michelle F Benoit, MD* \\ Gynecologic Oncology, Kaiser Permanente Bellevue Medical Center, USA
}

\begin{abstract}
Purpose: Gynecologic oncologists have different practice patterns based on practice setting and adjuvant treatments offered. Hospital and practice call coverage is not standardized and can vary widely as well. This study sought to: 1) Document the variety of practice settings and volume 2) Document the variety of support providers and their roles within these practice settings 3) Document vacation and CME time allocated 4) Document call coverage solutions.

Methods: A web based survey was distributed via email to 1005 U.S. registered full and candidate Society of Gynecologic Oncology (SGO) physician members in 2018. An electronic questionnaire consisting of 20 questions was distributed measuring different practice pattern variables (Appendix).

Results: A total of 1005 physicians were invited and 189 participated (18.9\%). $21.4 \%$ of respondents were in a single provider practice; $70.4 \%$ were divisionally located in an obstetrics/gynecology department, $17.6 \%$ were under an oncology center; $39.6 \%$ were academic based. $21.6 \%$ do not directly administer chemotherapy; $37.2 \%$ receive 2 weeks of $\mathrm{CME} ; 57.1 \%$ receive 4 weeks of vacation; $18.2 \%$ of persons who responded to the maternity leave question took 2 weeks off; $72.9 \%$ of persons who responded to the paternity leave question did not have leave available.

Conclusion(s): Results from this survey include descriptions of coverage and practice patterns that vary widely. This survey documents multiple practice patterns. It also documents options that are available and currently used for practice coverage for gynecologic oncology providers. Even though gynecologic oncology providers are considered subspecialty, the different components of the practice are still generalizable and multispecialty cross coverage is one solution. Hospital bylaws and practice coverage can be tailored to the current medical needs of the patient to include general or subspecialty surgery, medical oncology, internal medicine, and general or subspecialty gynecology cross coverage. Parental leave options were explored.
\end{abstract}

\section{Keywords}

Practice patterns, Parental leave, Call coverage, Hospital privileges, Chemotherapy, Advanced practice providers

\section{Introduction}

Gynecologic oncologists have different practice patterns within the United States. This is often based on the practice setting being academic versus community based. As more providers are transitioning from a private practice model to an employed physician model, and more fellows take their first job as an employed position, practice settings have changed widely in the last decade. SGO has reported practice patterns, the last results being distributed in 2015 [1]. The variety of practice settings has not been reported since, and data to support the changing climate of our practices has not been widely available. This survey was not affiliated with SGO and is an independent investigation.

This survey was distributed to evaluate the current state of practice settings. This includes provision of adjuvant therapy, the incorporation of advanced practice providers in the practice, call and call coverage options, the number of vacation and CME time allocated, and parental leave policies available and adopted.

\section{Materials and Methods}

\section{Survey design}

All candidate and full physician members of SGO who had a working email address listed in the SGO member directory were invited to participate in the study. The participation was elective and responses were anonymous. This web-based survey consisted of 20 questions. The survey included variables that represent different practice patterns, time off for vacation, $\mathrm{CME}$, and parental leave options. All data was self-re-

*Corresponding author: Michelle F Benoit, MD, Gynecologic Oncology, Kaiser Permanente Bellevue Medical Center, 11511 NE 10th St. Bellevue, WA 98004, USA, Tel: 425-510-1000, Fax: 425-510-3459

Accepted: June 17, 2019

Published online: June 19, 2019

Citation: Benoit MF (2019) Practice Patterns in Gynecology Oncology. Reports Gynecol Surg 2(1):18-21 
ported by survey respondents. All data was non-identifiable and contained no personal or medical information. There was no data capture outside of workparameters and allocated time off. Years in practice, post-fellowship, provider age, and other personal demographic information was not surveyed.

\section{Data collection}

Data was collected from February through July 2018. Results were captured anonymously. As there was no personal demographic or personal health information requested or evaluated, this review was deemed IRB exempt.

\section{Statistical analysis}

Clinical and practice data characteristics were reported descriptively using number and percent for categorical measurements. For continuous measurements, mean was used. Response rates are presented as frequencies and percentages. Results were sum tallied.

\section{Results}

One hundred eighty-nine physician providers participated in the survey. The average survey completion time was 1 minute 43 seconds. Some respondents participated in a portion of the study and did not complete specific questions, thus the denominator changed per each question and is reflective of this attrition.

Practice size was first investigated: $21.4 \%(40)$ of respondents were in a single provider practice; $24.1 \%(45)$ were in practice with one gynecologic oncology partner; $27.3 \%$ (51) were in a group of 3-4 gynecologic oncologists; $15.5 \%$ (29) were in a group of 5-6 partners; and $11.8 \%$ (22) were in a group more than 6 partners. Practice setting was explored next: $15.3 \%$ (17) were in private practice, $39.6 \%$ (44) were in an academic based setting; $33.3 \%$ were hospital employed physicians; $5.4 \%$ (6) were HMO based; and $6.3 \%$ (7) were in a multispecialty group.

Regardless of practice setting, divisional oversight was investigated next: $70.4 \%$ (76) of providers were housed in an obstetrics and gynecology department; $17.6 \%(19)$ were in the cancer center matrix; $6.5 \%$ (7) practiced within the surgery department; $1.9 \%$ (2) were housed in surgical oncology; and $3.7 \%$ (4) practiced in the medical oncology department. To ascertain surgical volume, the average number of cases per week was asked; $7.1 \%$ (8) of providers performed $<2$ major cases per week; $36.3 \%$ (41) performed 3-4 majors a week; $27.4 \%$ (31) performed 5-6 major surgeries a week; and 29.2\% (33) performed greater than 6 major surgeries per week. Scope of practice was evaluated with administration of chemotherapy as part of the practice setting: $78.4 \%$ (87) of physicians were identified to administer chemotherapy as part of their practice.

The geographic scope of coverage was examined and as surrogate, the number of hospitals covered was queried. Providers were found to cover only one hospital in $47.8 \%$ (54) of practices; $2-3$ hospitals in $43.4 \%$ (49) of practices; and 4-6 hospitals in $7.1 \%$ (8) of practices; $1.8 \%$ (2) of providers covered more than 6 facilities as part of their practice region.
With regards to clinic sites: $46.9 \%$ (53) of providers had one clinic site; $49.6 \%$ (56) had 2-3 clinic sites; and $2.7 \%$ (3) had 4-6 clinic sites they practiced out of. One provider respondent covered more than 6 clinics (0.88\%).

Resident assistance and teaching was identified to be part of $63.7 \%$ (72) of physicians' practices, where $36.3 \%$ (41) did not have any resident participation. Advanced practice practitioners (APP's) were found to be part of $79.5 \%$ (89) of participants'gynecologic oncology practices, whereas $20.1 \%$ (23) did not have APP's. APP's were identified to work in many roles within gynecologic oncology practices, and the sum total percent exceeds $100 \%$ representing this: $31.5 \%$ (29) provided surgical assistance; $58.7 \%$ (54) participated in chemotherapy administration; $41.3 \%$ (38) performed hospital rounding; $22.8 \%$ (21) took first call; and $93.5 \%$ (86) participated in clinics.

Call responsibilities were explored as well: $43.4 \%$ (49) of providers took their own weekday/weeknight call whereas $56.6 \%(64)$ had assigned call with a partner or coverage designee. Eighty-nine percent (99) of respondents were expected to have clinic or perform surgical cases on the postcall day, whereas $10.8 \%$ (12) had the post-call day designated for non-clinical activities. Forty-eight percent (54) of providers had scheduled administrative time whereas $52.2 \%$ (59) did not.

Time off for vacation and continuous medical education (CME) was reviewed: $16.1 \%$ (18) of providers had 3 weeks of vacation a year, $57.1 \%(64)$ had 4 weeks, $14.9 \%$ (16) had 5 weeks of vacation, and $12.5 \%$ (14) had 6 weeks of vacation built into their schedule yearly. The average vacation allocated was 4 weeks. Regarding CME, 37.2\% (42) of providers had 2 weeks of CME annually, $15 \%$ (17) had 3 weeks, and $6.2 \%$ (7) had 4 weeks, whereas $41.6 \%$ (47) had less than 2 weeks a year allocated. The average CME time was 1.9 weeks. Coverage for gynecologic oncology providers varied widely during their CME and vacation absences. The totals surpassed $100 \%$ so the data reveals that multiple provider specialties were tapped to cover the gynecologic oncology patients, likely based on the patient's presenting needs and phase in their treatment. Sixty-one percent (57) of gynecologic oncologists reported coverage from general gynecology for patients; urogynecology in $22.6 \%$ (21), general surgery covered $38.7 \%$ (36) of responding providers, surgical oncology in $32.3 \%$ (30), a hospitalist service in $16.1 \%(15)$, and medical oncology for $29 \%$ (27) of respondents. Compensation for call coverage was not provided $82.7 \%$ (91) of the time; $6.4 \%$ (7) of gynecologic oncology providers had some reciprocity; $3.6 \%$ (4) had lump sum financial compensation provided per day, $1.8 \%$ (2) per weekend, and $1.8 \%$ (2) per week.

Maternity and paternity leave were also investigated. Forty-four participants responded to the maternity leave question: $18.2 \%$ (8) of providers took 2 weeks off; $4.6 \%$ (2) took 3 and 4 weeks respectively; $2.3 \%$ (1) took 5 weeks; $20.5 \%$ (9) took 6 weeks; $16 \%$ (7) took 8 weeks; and 34.1\% (15) took 12 weeks. Fifty-nine persons responded to the paternity leave question, but for $72.9 \%$ (43) of these providers, paternity leave was not an option. Thirteen percent (8) took 1 week, 
$10.2 \%$ (6) took 2 weeks, and $1.7 \%$ (1) each took 3 and 6 weeks.

\section{Discussion}

Gynecologic oncologists were surveyed 4 years post the last SGO survey in this independent survey. They were found to have multiple types of practice settings. There is not one specific model that was found to be universal. Documentation of these survey results demonstrates various solutions used for call coverage, variations in CME and vacation time, as well as practice resource use and parental leave.

This survey documents that gynecologic oncologists are high volume surgeons. Ninety-three percent of providers have $>12$ major surgical cases per month. Volume greater than 10 surgeries a month has been denoted as high and some studies correlate higher volume with more successful outcomes [2]. Most gynecologic oncologists in this survey also have multidisciplinary practices, as almost $80 \%$ of respondents provide chemotherapy services.

A majority of practices are small consisting of 2 or fewer subspecialists (45\%). There is a high rate of burnout in the profession [3]. Time off for physician well-being and patient safety should be prioritized, but this comes with finding call coverage. Call coverage possibilities are demonstrated from this survey. Multiple other service lines were shown to provide coverage depending on the medical or surgical diagnosis and needs of the patient at that time-point in their oncology care [4]. Gynecologic oncologists can provide this data to their employers and to hospital bylaw committees. These entities may benefit from this additional information on current practice variables and it may guide them in denotation of practice setup and call coverage for subspecialty providers.

Parental leave policies should continue to be developed and offered to support our providers in their non-practice lives: $18.2 \%$ of respondents to the maternity leave question reported only taking 2 weeks of leave, and $73 \%$ of providers who responded to the paternity leave question said there was no option for any leave. At the author's current institution, gender neutral parental leave is being implemented and other institutions can follow in this lead. Non-medical organi- zations are also providing gender neutral parental leave policies which are helping to set new cultural expectations and norms [5].

Future evaluation of practice patterns can look at practice size stratified by practice setting to get a sense of practice size in academic versus community settings. Resident participation in the community practice setting would also be an interesting variable to evaluate given the high volume of surgeries that gynecologic oncologists perform. Residency programs may find these practice settings an additional resource to increase the surgical exposure for our trainees.

This survey investigation obtained strictly descriptive data. Limitations of this review include: Surveyed physicians reporting of their institutional practice patterns may be incorrect; there was a low response rate; some questions allowed multiple responses so the percentage response was over $100 \%$, and this survey data can provide no conclusions about the efficacy of specific practice patterns.

\section{Disclosures}

The author reports there is no conflict of interest.

\section{Funding Source}

The author reports there is no financial support for this project.

\section{References}

1. (2016) SGO State of the subspecialty survey report 2015.

2. Mowat A, Maher C, Ballard E (2016) Surgical outcomes for low-volume vs high-volume surgeons in gynecology surgery: A systemic review and meta-analysis. Am J Obstet Gynecol 215: 21-33.

3. Rath KS, Huffman LB, Phillips GS, et al. (2015) Burnout and associate factors among members of the Society of Gynecologic Oncology. Am J Obstet Gynecol 213: 824.e1-824.e9.

4. Temkin SM (2019) Personal reflections on gynecologic oncology and its future. Obstet Gynecol 133: 1255-1258.

5. https://www.hrdive.com/news/jpmorgan-chase-pays-5m-tosettle-dads-parental-leave-suit/556024. 


\section{Appendix}

\section{Survey as distributed.}

Question 1:

How many physicians are in your practice: single, 2-3, 4-6, > 6 .

Question 2:

What is your practice setting: private, academic, hospital employed, HMO, multispecialty.

\section{Question 3:}

Do you administer chemotherapy in your practice: yes, no.

Question 4

How many CME weeks are you allocated per year: 2, 3, 4.

Question 5:

How many vacation weeks are you allocated per year: 3, 4, 5, 6 .

Question 6:

Who covers your practice during $\mathrm{CME} /$ vacation (given that partners do attend same meetings occasionally and if have resident/fellow they have to have assigned backup): gynecology, gynecologic oncology, general surgery, surgical oncology, medical oncology, hospitalist, urogynecology, reproductive endocrinology and infertility (check all that apply).

Question 7:

Is there compensation for call coverage: reciprocity, lump sum, per patient, per day, per week, per weekend, none.

Question 8:

What is your week-night call? You take: all of your own call, assigned call.

\section{Question 9:}

What is your average surgical volume for major procedures per week: $2,3-5,6+$.
Question 10:

How many hospitals do you cover: 1, 2-3, 4-6, > 6 .

Question 11:

How many clinic sites do you cover: $1,2-3,4-5,>6$.

Question 12:

Do residents participate in your practice: yes, no.

Question 13:

Do you have an Advanced Practice Provider (APP) as part of your team (PA-C, NP, etc.): yes, no.

Question 14:

What is the role of the APP in your practice: surgical assist, chemotherapy assist, rounding, call backup, first call (check all that apply).

Question 15:

Under what umbrella/department is your practice: obstetrics and gynecology, oncology, surgery, surgical oncology, medical oncology, solo.

Question 16:

Do you have scheduled administrative time: yes, no.

Question 17:

Are you expected to have clinic or operate on your post-call day: yes, no.

Question 18:

How many weeks did you take for maternity leave (if applicable): 1, 2, 3, 4, 5, 6, NA.

Question 19:

How many weeks did you take for paternity leave (if applicable): 1, 2, 3, 4, 5, 6, NA.

Question 20: Comments.

DOI: $10.36959 / 909 / 467$ 